Artículo original

\title{
Complicaciones en sitio de canulación en pacientes con oxigenación con membrana extracorpórea veno-arterial en una institución de Medellín
}

\section{Complications at the cannulation site in patients with arteriovenous extracorporeal membrane oxygenation in an institution in Medellín}

María Fernanda Gómez Gil ${ }^{1 凶} \underline{\mathrm{ORCID}}$, Catalina Marín Tangarife $2 \llbracket \underline{\mathrm{ORCID}}$, Bibiana Milena Serna Monsalve ${ }^{3 凶} \underline{\mathrm{ORCID}}$

${ }^{1}$ Hospital universitario de San Vicente fundación.

2 Clínica Cardiovid.

${ }^{3}$ Clínica SOMA.

Fecha correspondencia:

Recibido: 17 octubre 2021.

Aceptado: 30 noviembre 2021.

Forma de citar:

Gómez-Gil M, Marín-Tangaerife

C, Serna-Monsalve B.

Complicaciones en sitio de canulación en pacientes con oxigenación con membrana extracorpórea veno-arterial en una institución de Medellín. Rev CES Enf [Internet]; 2(2):28-39. Disponible en: https://dx.doi.org/ $\underline{10.21615 / c e s e n f e r m .6441}$

\section{Open access}

C) Derecho de autor

Licencia creative commons

Ética de publicaciones

Revisión por pares

Gestión por Open Journal System DOI: $10.21615 /$ cesenferm.6441

ISSNe: $2745-049 \mathrm{X}$

\section{Resumen}

El soporte con terapia ECMO es una técnica utilizada para dar soporte a pacientes críticamente enfermos. Se ha venido desarrollando a lo largo de los años, en la actualidad se conocen detalladamente las complicaciones más frecuentes que se pueden presentar, pero es necesario publicaciones enfocadas a las complicaciones en piel relacionadas con el sitio de canulación. Objetivo: identificar factores clínicos, personales y de estancia hospitalaria relacionados con el desarrollo de complicaciones en piel en pacientes soportados con ECMO veno-arterial con canulación femoral, con el fin de proponer acciones preventivas en el manejo. Metodología: estudio retrospectivo, comparativo de tipo observacional de 51 registros de historias clínicas de pacientes sometidos a soporte con terapia ECMO veno-arterial con canulación femoral en una institución de alto nivel de complejidad en la ciudad de Medellín entre agosto de 2015 y enero de 2020. Se analizaron variables demográficas, comorbilidades, uso de medicamentos, tiempo con el soporte y días de estancia hospitalaria Se contó con aprobación del comité 

de los pacientes, siendo infección la más frecuente, con factores relacionados a su aparición como mayor tiempo transcurrido con el soporte, hipertensión arterial y uso de inmunosupresores, lo cual generó aumento en los días de estancia hospitalaria. Conclusión: La infección en el sitio de canulación fue la complicación más frecuente, y se relaciona con una mayor edad, la HTA, la administración de inmunosupresores y mayor tiempo de soporte con la terapia.

Palabras clave: ECMO; piel; cicatrización; hipertensión.

\section{Abstract}

ECMO therapy support is a technique used to support critically ill patients. It has been developed over the years. At present, the most frequent complications that may occur are known in detail, but publications focused on complications in the skin related to the cannulation site are necessary. Objective: to identify clinical, personal, and hospital stay factors related to the development of skin complications in patients supported with arteriovenous ECMO with femoral cannulation to propose preventive actions in management. Methodology: a retrospective, comparative observational study of 51 records of medical records of patients undergoing support with arteriovenous ECMO therapy with femoral cannulation in a highly complex institution in the city of Medellín between August 2015 and January 2020 Demographic variables, comorbidities, use of medications, time with support and days of hospital stay were analyzed with the approval of the institution's ethics committee. Results: complications in $20 \%$ of patients, infection being the most frequent, with factors related to its appearances such as longer time elapsed with support, arterial hypertension, and use of immunosuppressants, which generated an increase in the days of hospital stay. Conclusion: Infection at the cannulation site was the most frequent complication, and it is related to older age, hypertension, administration of immunosuppressants, and longer support time with therapy.

Keywords: ECMO; skin; wound healing; hypertension.

\section{Introducción}

El nacimiento de la Extracorporeal membrane oxygenation (ECMO) se remonta a 1939 con el desarrollo de la primera máquina corazón-pulmón por John Gibbon, lo que culminó en 1953 con la primera operación a corazón abierto ${ }^{(1)}$. Debido a la alta tasa de mortalidad de los pacientes con Síndrome de dificultad respiratoria agudo (SDRA) en ese momento, estos 
primeros informes evocaron un gran interés y fueron el impulso para el primer ensayo clínico aleatorizado multicéntrico de ECMO prolongado para adultos con SDRA. Varios investigadores continuaron estudiando y mejorando la técnica ${ }^{(1)}$.

La terapia ECMO se realiza a través de una máquina que reemplaza la función del corazón y los pulmones para los pacientes hospitalizados en las Unidades de cuidado intensivo (UCI); con una duración desde pocas horas hasta días, pero en general puede ser necesaria por pocas semanas dependiendo del progreso de su condición. Este soporte es de gran utilidad pues da asistencia al paciente mientras logra la recuperación de afecciones potencialmente mortales como lo son la insuficiencia cardiorrespiratoria aguda, miocarditis aguda, intoxicación con agentes depresores cardíacos o síndrome coronario con shock cardiogénico ${ }^{(2)}$.

Hay dos tipos básicos de soporte ECMO: oxigenación por membrana extracorpórea venovenoso (ECMO-VV) que proporciona solo soporte respiratorio y oxigenación por membrana extracorpórea veno-arterial (ECMO-VA), el cual soporta tanto el corazón como los pulmones y, por lo tanto, es el apropiado para pacientes con Insuficiencia cardiaca ${ }^{(2)}$.

En el ECMO-VA se puede hacer una canulación central, en la cual la sangre se drena desde la aurícula derecha y regresa a la parte proximal de la aorta. Con la canulación periférica, la sangre se drena de las grandes venas proximales a través de una vena femoral o yugular, utilizando una incisión quirúrgica, y se devuelve a la aorta mediante la canulación de una arteria, ya sea la carótida, axilar o femoral ${ }^{(3)}$. Este procedimiento requiere que se realice una incisión en el sitio, donde se introduce una cánula que trae consigo la alteración en la integridad cutánea con la posible afección de tejidos más profundos ${ }^{(4)}$.

La cicatrización se da mediante un grupo de procesos interactivos que se superponen en el tiempo y que comienzan cuando la piel recibe una agresión en cualquiera de sus capas. Estos procesos involucran múltiples factores de crecimiento y proliferación celular, los cuales pueden verse afectados por múltiples factores como el tabaquismo ${ }^{(5,6)}$, el desequilibrio nutricional, tanto por defecto como por exceso ${ }^{(7,8)}$, la inmunosupresión ${ }^{(9)}$ y comorbilidades como la diabetes mellitus ${ }^{(10)}$, generando esto retraso en la cicatrización y consigo la aparición de complicaciones relacionadas con la herida ${ }^{(11)}$.

Se han descrito complicaciones asociadas a la terapia ECMO como trombosis y sangrado, siendo este último el que más contribuye a la muerte de pacientes sometidos a dicho soporte ${ }^{(12)}$. También se ha descrito las infecciones como complicación en entre el $10-12 \%$ en población 
pediátrica y en el $21 \%$ de adultos, tanto en el torrente sanguíneo cómo en el sitio de canulación, entre otros ${ }^{(13)}$. La infección del sitio quirúrgico se relaciona con una intervención quirúrgica, ya sea en el propio lecho quirúrgico o en su proximidad, ocurre dentro de los 30 días tras la cirugía y se clasifican en superficiales, profundas y de órgano espacio ${ }^{(14)}$. La infección del sitio operatorio en el sitio de las cánulas esta reportado alrededor de $10 \%{ }^{(13)}$.

El objetivo del estudio fue identificar los factores clínicos, personales y de estancia hospitalaria relacionados con el desarrollo de complicaciones en piel en pacientes soportados con ECMOVA periférico con canulación femoral en una institución de alta complejidad de la ciudad de Medellín.

\section{Metodología}

Se realizó un estudio retrospectivo, comparativo de tipo observacional. La muestra se constituyó con los 51 pacientes que fueron sometidos a terapia con ECMO-VA con canulación periférica entre agosto de 2015 y enero de 2020 en las UCls de la Institución. La información se obtuvo de las historias clínicas, previo a la aprobación del Centro de investigaciones de la clínica.

Se realizó un filtro inicial en las bases de datos de la Clínica detectando los pacientes que tuvieron soporte con terapia ECMO-VA periférico con canulación femoral en el periodo establecido. Posteriormente se realizó un análisis documental al extraer la información de las historias clínicas electrónicas y se realizó búsqueda de las siguientes variables: características demográficas (sexo y edad), comorbilidades (hipertensión arterial, insuficiencia renal, insuficiencia cardiaca), uso de medicamentos durante la terapia ECMO (antibióticos, vasoactivos e inmunosupresores), antecedentes personales (tabaquismo), tiempo de duración del soporte con la terapia ECMO y tiempo de estancia hospitalaria.

Los datos fueron recolectados y registrados en un archivo de Excel, posteriormente se analizaron en el programa estadístico EPIDAT 4.2. Se calcularon porcentajes y el valor de p para todas las variables mencionadas anteriormente. La presente investigación se clasifica como sin riesgo, según la Resolución 8430 de $1993{ }^{(15)}$. En todo momento se mantuvo la confidencialidad de la información recolectada para la realización del estudio.

\section{Resultados}

De los 51 pacientes tuvieron soporte con terapia ECMO-VA periférico con canulación femoral durante el tiempo del estudio, $20 \%$ presentaron complicaciones relacionadas al sitio de canulación femoral. Del total de la muestra $68,6 \%$ fueron de sexo masculino y $31,3 \%$ de sexo 
femenino, $60 \%$ de quienes tuvieron complicaciones fueron de sexo masculino y $40 \%$ de sexo femenino. De otro lado, el promedio de edad del total de la muestra fue de 46 años, mientras que el promedio de edad en la cuales se presentaron las complicaciones fue de 51 años.

Entre los antecedentes patológicos importantes se encontró hipertensión arterial (HTA) en 60\% pacientes con complicación y $39 \%$ pacientes sin complicación. HTA con falla cardiaca en $30 \%$ de los pacientes que presentaron complicación y $17,0 \%$ de quienes no la presentaron. El $20 \%$ de los pacientes padecían HTA, falla cardiaca y falla renal concomitantemente y presentaron complicaciones, mientras que el 7,3\% las padecían también y no presentaron complicaciones. De quienes presentaron complicaciones $10 \%$ tenía falla cardiaca y falla renal, $2,4 \%$ tenía los mismos antecedentes y no presento complicación.

Entre los medicamentos que recibían los pacientes se encontró que $60 \%$ de quienes presentaron complicación recibían inmunosupresores, en contraste con $31,7 \%$ que no presentaron complicación y también los recibían. De quienes recibían antibióticos junto con inmunosupresores $40 \%$ presentaron complicación y $17 \%$ no presentaron. Se encontró que de quienes presentaron complicación 30,0\% tenían antecedente de tabaquismo.

De otro lado, se observó una diferencia significativa respecto al tiempo con el soporte, observándose que quienes sufrieron complicación tuvieron un promedio de 13 días con el soporte, mientras que, quienes no presentaron complicación tuvieron un promedio de 8,5 días de soporte con la terapia $(p=0,035)$

Se evidenció que la estancia hospitalaria de quienes no tuvieron complicación tuvo un promedio de 67,2 días mientras que quienes no presentaron complicación tuvieron un promedio de estancia de 37,6 días, pero esta aparente diferencia no fue significativa $(p=0.056$. Estos resultados se muestran en la tabla 1.

Las complicaciones que se encontraron en el sitio de canulación femoral en los pacientes con soporte de terapia ECMO-VA periférico fueron infección del sitio de canulación en $70 \%$ pacientes, dehiscencia en $20 \%$ y una combinación de las anteriores en $1 \%$. Los resultados de pueden apreciar en la tabla 2. 
Tabla 1. Características asociadas con la aparición de complicaciones en pacientes sometidos a terapia con ECMO-VA con canulación periférica femoral.

\begin{tabular}{|c|c|c|c|c|c|}
\hline \multirow[t]{2}{*}{ Variable } & \multicolumn{4}{|c|}{ Complicación } & \multirow[b]{2}{*}{ Valor $p$} \\
\hline & SI & $\%$ & NO & $\%$ & \\
\hline \multicolumn{6}{|l|}{ Sexo } \\
\hline Masculino & 6 & $60 \%$ & 29 & $70,7 \%$ & \\
\hline Femenino & 4 & $40 \%$ & 12 & $29,2 \%$ & 0,512 \\
\hline \multicolumn{6}{|l|}{ Edad } \\
\hline$x$ & 51,1 & & 45 & & \multirow{4}{*}{0,267} \\
\hline DS & 13,4 & & 17,3 & & \\
\hline Me & 53 & & 49 & & \\
\hline RIQ & 16 & & 24,5 & & \\
\hline \multicolumn{6}{|l|}{ Comorbilidades } \\
\hline Ningún antecedente personal & 3 & $30 \%$ & 14 & $34,1 \%$ & 0,803 \\
\hline Hipertensión arterial & 6 & $60 \%$ & 16 & $39,0 \%$ & 0,230 \\
\hline Falla cardiaca & 6 & $60 \%$ & 21 & $51,2 \%$ & 0,618 \\
\hline Hipertensión arterial/falla cardiaca & 3 & $30 \%$ & 7 & $17,0 \%$ & 0,356 \\
\hline $\begin{array}{l}\text { Hipertensión arterial/falla cardiaca/falla } \\
\text { renal }\end{array}$ & 2 & $20 \%$ & 3 & $7,3 \%$ & 0,227 \\
\hline Falla cardiaca y falla renal & 1 & $10 \%$ & 1 & $2,4 \%$ & 0,269 \\
\hline \multicolumn{6}{|l|}{ Días ECMO } \\
\hline $\mathrm{x}$ & 13 & & 8,5 & & \multirow{4}{*}{$0,035^{*}$} \\
\hline DS & 5,1 & & 5 & & \\
\hline Me & 13,5 & & 7 & & \\
\hline RIQ & 10 & & 8,5 & & \\
\hline \multicolumn{6}{|l|}{ Estancia } \\
\hline$x$ & 67,2 & & 37,6 & & \multirow{4}{*}{$0,056^{*}$} \\
\hline DS & 39,2 & & 31,7 & & \\
\hline $\mathrm{Me}$ & 57 & & 28 & & \\
\hline RIQ & 82 & & 38 & & \\
\hline \multicolumn{6}{|l|}{ Tratamiento farmacológico } \\
\hline Vasoactivos & 3 & $30 \%$ & 21 & $51,2 \%$ & 0,228 \\
\hline Inmunosupresores & 6 & $60 \%$ & 13 & $31,7 \%$ & 0,097 \\
\hline Vasoactivos y antibióticos & 1 & $10 \%$ & 12 & $29,2 \%$ & 0,210 \\
\hline $\begin{array}{l}\text { Antibióticos/vasoactivos/ } \\
\text { inmunosupresores }\end{array}$ & 2 & $20 \%$ & 6 & $14,6 \%$ & 0,676 \\
\hline Antibióticos/inmunosupresores & 4 & $40 \%$ & 7 & $17,0 \%$ & 0,114 \\
\hline Antecedente de Tabaquismo & 3 & $30 \%$ & 11 & $26,8 \%$ & 0,840 \\
\hline
\end{tabular}

Tabla 2. Complicaciones en piel en pacientes sometidos a terapia ECMO-VA asociadas al sitio de canulación periférica femoral.

\begin{tabular}{lcc}
\multicolumn{1}{c}{ Complicación } & $\boldsymbol{n = 5 1}$ & $\mathbf{\%}$ \\
\hline Infección & 7 & $13,7 \%$ \\
\hline Dehiscencia & 2 & $3,9 \%$ \\
\hline Infección/dehiscencia & 1 & $1,9 \%$
\end{tabular}




\section{Discusión}

Este estudio identificó los factores clínicos, personales y de estancia hospitalaria relacionados con el desarrollo de complicaciones en piel en pacientes soportados con ECMO-VA periférico con canulación femoral, encontrándose la infección en el sitio de canulación como la complicación más frecuente, la cual se relaciona con un mayor número de días del soporte, ser del sexo masculino, edad superior a los 50 años, presencia de HTA y el uso de inmunosupresores. Todo lo anterior, adicionalmente generó un aumento de la estancia hospitalaria.

La infección en el sitio de canulación, como principal complicación ya ha sido descrita en la literatura científica. En Francia, Allou et al ${ }^{(16)}$ evidenciaron un $17,7 \%$ de infección relacionada con la cánula, mientras que Schmidt et al ${ }^{(17)}$ un $10 \%$, Hahne et al ${ }^{(18)}$ en Alemania y Sun et al ${ }^{(19)}$ en Taiwán reportaron un 15\% y 3,5\% respectivamente. Los resultados anteriores coinciden con los de este estudio y muestran la importancia de esta complicación en pacientes sometidos a ECMO-VA.

Hay una gran relación entre los días de terapia ECMO y las complicaciones, pues el promedio de días entre quienes las presentaron y quienes no las presentaron una diferencia significativa de 5 días entre ambos grupos. Es conocido que el tiempo de hospitalización y de invasión es directamente proporcional a la aparición de complicaciones relacionadas con el cuidado de la salud, las infecciones nosocomiales son uno de los mayores problemas en las unidades de cuidado intensivo y son causantes de gran morbilidad y mortalidad en los pacientes que las padecen ${ }^{(20)}$.

Como menciona Grau ${ }^{(21)}$, la estancia hospitalaria es una de las variables más sensibles relacionada con el aumento de costos en atención en salud y en muchos de los casos está relacionado con las infecciones y el tiempo que conlleva su tratamiento, pues requiere mayor tiempo de cuidados de alta complejidad.

Asimismo, el estudio muestra que las complicaciones se presentaron en mayor proporción en pacientes que estaban en un promedio de edad mayor (51 años). Este aspecto ha sido documentado como uno relevante en el proceso de cicatrización, ya que a mayor edad la piel tiene una pérdida progresiva de sus funciones por el efecto de factores intrínsecos y extrínsecos del envejecimiento ${ }^{(22)}$. También, histológicamente las cicatrices son diferentes en niños y en adultos $^{(23)}$ lo que refuerza el efecto de la edad como factor relevante en dicho proceso. 
El antecedente patológico que más se relacionó con la aparición de complicaciones fue la HTA, ya fuera sola o en conjunto con otras patologías. La HTA prolongada trae consigo complicaciones vasculares por el aumento de la resistencia vascular en los vasos (24); así como otras complicaciones como infarto agudo al miocardio, insuficiencia renal, accidente cerebrovascular, insuficiencia renal y arteriopatía periférica ${ }^{(25)}$, esta última asociada a lesiones de difícil cicatrización.

La inmunosupresión inducida por medicamentos se observa en receptores de trasplantes y otras condiciones, causando disminución de la respuesta inflamatoria y por consiguiente afecta la cicatrización de las heridas al retrasar la síntesis de proteínas y la proliferación celular ${ }^{(9)}$. Los inmunosupresores actuales tienen gran efectividad en el éxito de los trasplantes, pero la ausencia de respuesta inmunitaria se da es a nivel general ${ }^{(26)}$ motivo por el cual pacientes con dicho tratamiento farmacológico tienen mayor riesgo de contraer infecciones que quienes no lo reciben. Entre los medicamentos que recibían los pacientes que tuvieron complicaciones destaca el uso de inmunosupresores.

\section{Conclusiones}

La infección en el sitio de canulación fue la complicación más frecuente, y se relaciona con una mayor edad, la HTA, la administración de inmunosupresores y mayor tiempo de soporte con la terapia. Dichas complicaciones generaron una estancia hospitalaria más prolongada.

Se hace necesario revisar y evaluar los cuidados de enfermería que se brindan a los pacientes y en especial, en el sitio de canulación. Asimismo, realizar intervenciones enfocadas a mantener el sitio de canulación con baja carga bacteriana con en uso de agentes tópicos y apósitos antimicrobianos. El desarrollo de intervenciones educativas basadas en estos resultados y en otros también es recomendable.

Como limitaciones para el estudio podemos mencionar el reducido número de pacientes de la muestra y la realización del estudio en una sola institución de la región antioqueña. Sin embargo, los resultados pueden ser generalizables a otras instituciones hospitalarias colombianas de características similares. Asimismo, estos resultados aportan al escaso conocimiento actual sobre las complicaciones asociadas al sitio de canulación en pacientes con terapia ECMO-VA, por lo que además, pueden ser un punto de partida para futuras investigaciones de mayor alcance y profundidad. 
Julio - diciembre de 2021

\section{Conflicto de intereses}

Las autoras no refieren conflicto de intereses.

\section{Fuentes de financiación}

Para la realización del presente estudio no se tuvo ninguna fuente de financiación.

\section{Referencias}

1. Patroniti N. Extracorporeal Support of Gas Exchange [Internet]. ClinicalKey. 2021 [citado 15 abril 2021]. Disponible en: https://www-clinicalkeyes.ces.idm.oclc.org/\#!/content/book/3-s2.0B9781455733835001032?scrollTo=\%23hl0000742.

2. Le Gall A, Follin A, Cholley B, Mantz J, Aissaoui N, Pirracchio R. «Veno-Arterial-ECMO in the Intensive Care Unit: From Technical Aspects to Clinical Practice [Internet]. ScienceDirect. 2018 [citado 29 octubre 2021]. Disponible en: https://doi.org/10.1016/i.accpm.2017.08.007.

3. Lafçı G, Baran A, Ümit A, Faruk O. Use of Extracorporeal Membrane Oxygenation in Adults Support of Gas Exchange [Internet]. heartlungcirc.org. 2013 [citado 18 abril 2021]. Disponible en: https://doi.org/10.1016/i.hlc.2013.08.009.

4. Chilcott M. Wound Dressing [Internet]. ClinicalKey. 2020 [citado 2 septiembre 2021]. Disponible en: https://clinicalkey.cesproxy.elogim.com/\#!/content/book/3-s2.0B9780323476331000338?scrollTo=\%23hl0000398.

5. Gill J, Yu S, Neuhaus I. Tobacco smoking and dermatologic surgery [Internet]. ClinicalKey. 2013 [citado 15 abril 2021]. Disponible en:. https://www-clinicalkeyes.ces.idm.oclc.org/\#!/content/journal/1-s2.0-S0190962212010250.

6. Suzuki J, Resnik R. Dehiscencia de la herida: apertura de la línea de incisión [Internet]. ClinicalKey. 2018 [citado 2 junio 2021]. Disponible en:. https://clinicalkey.cesproxy.elogim.com/\#!/content/book/3-s2.0B9788491132721000113.

7. Seitz A. Wound Healing [Internet]. ClinicalKey. 2018 [citado 29 octubre 2021]. Disponible en: $\quad$ https://clinicalkey.cesproxy.elogim.com/\#!/content/book/3-s2.0B9780323529808000044?scrollTo=\%23hl0000238. 
8. Jacob B, Pierce K. Tratamiento de la hernia inguinal recidivante [Internet]. ClinicalKey. 2020 [citado 29 octubre 2021]. Disponible en: https://clinicalkey.cesproxy.elogim.com/\#!/content/book/3-s2.0B9788491137443001173?scrollTo=\%23hl0000116.

9. $\mathrm{Hu} \mathrm{M}$, Zielins $\mathrm{E}$, Longaker $\mathrm{M}$, Lorenz $\mathrm{H}$. Scar prevention, treatment, and revision [Internet]. ClinicalKey. 2018 [citado 13 septiembre 2021]. Disponible en: https://clinicalkey.cesproxy.elogim.com/\#!/content/book/3-s2.0B978032335694700014X?scrollTo=\%23hl0000565.

10. Leong M, Murphy K, Phillips L. Cicatrización de las heridas [Internet]. ClinicalKey. 2018 [citado 29 octubre 2021]. Disponible https://clinicalkey.cesproxy.elogim.com/\#!/content/book/3-s2.0B9788491131328000068?scrollTo=\%23hl0001373.

11. Beyene R, Derryberry S, Barbul A. The Effect of Comorbidities on Wound Healing [Internet]. ClinicalKey. 2020 [citado 7 mayo 2021]. Disponible en: https://wwwclinicalkey-es.ces.idm.oclc.org/\#!/content/journal/1-s2.0-\$0039610920300475.

12. Murphy DA, Hockings LE, Andrews RK, Aubron C, Gardiner EE, Pellegrino VA, Davis AK. Extracorporeal membrane oxygenation-hemostatic complications. Transfus Med Rev. 2015 Apr;29(2):90-101. doi: 10.1016/j.tmrv.2014.12.001. Epub 2014 Dec 18. PMID: 25595476.

13. Estrada V, Cortes L, Pedroza S, Camargo T. Infecciones asociadas con la terapia con Membrana de Oxigenación Extracorpórea en pacientes adultos. Rev Perfusión Colombia (1 - N.o 2) año 2018 pág. 54-60.

14. Fisterra. Infecciones en cirugía [Internet]. ClinicalKey. 2020 [citado 13 septiembre 2021]. Disponible en: https://clinicalkey.cesproxy.elogim.com/\#!/content/guides techniques/52-s2.0mt fis 2563

15. Ministerio de Salud. Resolución 8430 de 1993. [Internet]. [citado 1 mayo 2021]. Disponible en: https://www.minsalud.gov.co/sites/rid/Lists/BibliotecaDigital/RIDE/DE/DIJ/ 
16. Allou N, Lo Pinto H, Persichini R, Bouchet B, Braunberger E, Lugagne N, et al. CannulaRelated Infection in Patients Supported by Peripheral ECMO: Clinical and Microbiological Characteristics [Internet]. ClinicalKey. 2019 [citado 5 septiembre 2021]. Disponible en: https://doi.org/10.1097/MAT.0000000000000771.

17. Schmidt M, Bréchot N, Hariri S, Guiguet M, Luyt CE, Makri R, Leprince P, Trouillet JL, Pavie A, Chastre J, Combes A. Nosocomial Infections in Adult Cardiogenic Shock Patients Supported by Venoarterial Extracorporeal Membrane Oxygenation [Internet]. Pub Med. 2012 [citado 5 septiembre 2021]. Disponible en: https://pubmed.cesproxy.elogim.com/22990851/

18. Hahne K, Horstmann C, Fischer D, Köck R, Peters G, Lebiedz P. Cannula-Related Infection in Adult Medical Intensive Care Unit Patients Undergoing Extracorporeal Life Support and Extracorporeal Membrane Oxygenation [Internet]. The Journal of Hospital Infection 2015 [citado 6 septiembre 2021]. Disponible en: https://doi.org/10.1016/i.jhin.2015.08.022.

19. Sun HY, Ko WJ, Tsai PR, Sun CC, Chang YY, Lee CW, Chen YC. Infections occurring during extracorporeal membrane oxygenation use in adult patients. [Internet]. Ovid 2010 [citado 12 septiembre2021]. Disponible en: https://oce.ovid.com/article/00005362$\underline{201011000-00026 .}$

20. Zaragoza R, Ramirez P, Lopez-Puello M. Infección nosocomial en las unidades de cuidados intensivos. [Internet]. ClinicalKey 2014 [citado 12 septiembre 2021]. Disponible en: $\quad$ https://clinicalkey.cesproxy.elogim.com/\#!/content/journal/1-s2.0$\underline{\text { S0213005X14000597. }}$

21. Grau S. Principales variables que determinan el coste económico en el tratamiento de una infección [Internet] ClinicalKey 2017 [citado 2 septiembre 2021].Disponible en: https://www-clinicalkey-es.ces.idm.oclc.org/\#!/content/journal/1-s2.0S0213005X17300356.

22. Boukovalas S, Aliano K, Phillips L, Norbury W. Wound Healing [Internet]. ClinicalKey 2021 [citado 29 octubre 2021]. Disponible en: https://www-clinicalkeyes.ces.idm.oclc.org/\#!/content/book/3-s2.0B9780323640626000062?scrollTo=\%23hl0001620.

23. Westra I et al. Investigating Histological Aspects of Scars in Children. [Internet] PubMed 2017 [citado 1 septiembre 2021] Disponible en: https://doi.org/10.12968/jowc.2017.26.5.256. 
24. Restrepo J, Patiño M, y Rojas J. Cuidado del paciente con heridas: de lo básico a lo avanzado. 1 ed. CIB, p177-178; 2020.

25. Graham T. Hypertension [Internet] ClinicalKey. 2021 [citado 2 de septiembre de 2021]. Disponible en: https://clinicalkey.cesproxy.elogim.com/\#!/content/book/3-s2.0B9780323790062000306?scrollTo=\%23hl0000814.

26. Adams A, Ford M, Larsen P. Inmunobiología de los trasplantes e inmunodepresión [Internet] ClinicalKey 2017 [Accedido 2 septiembre 2021] Disponible en: https://wwwclinicalkey-es.ces.idm.oclc.org/\#!/content/book/3-s2.0-B978849113132800024X. 